\title{
SINTOMATOLOGÍA TRACTO URINARIO INFERIOR Y DISFUNCIÓN ERÉCTIL
}

\author{
Luis López-Fando Lavalle, Antonio Allona Almagro, Carolina Bueno Bravo y Luis Córdoba \\ Martínez.
}

Servicio de Urología. Hospital Ramón y Cajal. Madrid. España.

\begin{abstract}
Resumen.- La relación entre los síntomas del tracto urinario inferior (STUI) y la disfunción eréctil (DE) es el resultado de su mayor asociación en edades avanzadas. Sin embargo, varias investigaciones demuestran que los síntomas urinarios se relacionan en forma independiente con disfunción sexual y menor satisfacción. Asimismo, la gravedad de los STUI se correlaciona con la magnitud de la disfunción sexual en todos los grupos etarios, lo cual sugiere una posible relación causal.
\end{abstract}

Una serie de hipótesis se han formulado para explicar la existencia de una fisiopatología común entre STUI y DE. En la actualidad, esta relación entre STUI y DE está apoyada por cuatro teorías, no mutuamente excluyentes, que incluyen (a) la hiperactividad autonómica y la hipótesis de síndrome metabólico, (b) los cambios en la sintetasa de óxido nítrico / óxido nítrico (NOS / $\mathrm{NO}$ de la vía guanina monofosfatasa en la próstata y el pene, (c) la activación de Rho-kinasa y la vía de la endotelina, y (d) las consecuencias fisiopatológicas de la aterosclerosis pélvica.

Dada la contribución del funcionamiento sexual en el mantenimiento de la calidad de vida, en la elección del tratamiento para el paciente con hiperplasia prostática benigna deben tomarse en cuenta los posibles efectos negativos sobre la función sexual.

El enfoque terapéutico combinado de estas dos entidades (DE y STUI) provoca un beneficio sobre el paciente tanto en la sintomatología urinaria como en la esfera sexual, aunque se precisan estudios controlados con placebo para confirmar estos datos y dilucidar el papel de la terapia de combinación para el tratamiento de estas dos condiciones.

Palabras clave: Tracto urinario inferior. Síntomas. Disfunción eréctil.

Summary.- OBJECTIVES: The relationship between lower urinary tract symptoms (LUTS) and erectile dysfunction (ED) is the result of their greater association in advanced age. Nevertheless, several investigations show that urinary tract symptoms have an independent relationship with sexual dysfunction and lower satisfaction. Likewise, the severity of LUTS correlates with the magnitude of sexual dysfunction in all age groups, which suggests a possible causal relationship.

A series of hypothesis have been posed to explain the existence of a common physiopathology for LUTS and ED. Currently, this relationship between LUTS and ED is
Luis López-Fando Lavalle Hospital Ramón y Cajal Carretera de Colmenar Km 9, 100 28034 Madrid (España).

Ilfando@hotmail.com 
supported on four theories, which are not mutually excluding, (a) autonomic hyperactivity and metabolic syndrome hypothesis, (b) changes in nitric oxide/nitric oxide (NOS/NO) synthetase in the guanine monophosphatase pathway in penis and prostate, (c) the activation of Rho kinase and the endothelin pathway, and (d) the physiopathological consequences of pelvic arteriosclerosis. Given the contribution of sexual function to keep the quality of life, possible negative effects on sexual function should be taken into consideration when choosing treatment for benign prostatic hyperplasia.

The combined therapeutic approach of these two entities (ED and LUTS) brings a benefit to the patient both in urinary symptoms and sexual sphere, although placebo controlled studies are required to confirm these data and to ascertain the role of combination therapy in the treatment of both conditions.

Keywords: Lower urinary tract. Symptoms. Erectile dysfunction.

\section{INTRODUCCIÓN}

La prevalencia de síntomas del tracto urinario inferior (STUI) asociados con hiperplasia prostática benigna (HPB) en hombres de edad madura y ancianos es importante y está aumentando con el envejecimiento de las sociedades occidentales. La disfunción eréctil (DE) en el paciente con STUI, ha sido motivo de investigación dada la asociación de estos dos problemas en el varón por encima de los 40 años de edad. Numerosos estudios han sido publicados correlacionando estos dos problemas mediante índices estandarizados de uso frecuente en la consulta de urología como lo son la escala Internacional de Síntomas Prostaticos (IPSS) y el Índice Internacional de Función Erectil (IIEF). La intensidad de los STUI constituye para algunos autores un factor independiente de disfunción sexual (trastornos de erección y de eyaculación). Las respuestas eréctiles y eyaculatorias anómalas pueden tener un efecto negativo notable en la calidad de vida, con niveles crecientes de ansiedad y depresión. Por lo tanto, la preservación de la función sexual debe ser considerada en el tratamiento de estos pacientes.

En este trabajo se realizara una revisión exhaustiva de la bibliografía publicada hasta la fecha para aclarar varios aspectos fundamentales en el conocimiento y manejo de ambos problemas y como las opciones terapéuticas actuales permiten mejorar la sintomatología relacionada con la HBP así como tratar al mismo tiempo la DE. Para la comprensión de cómo estos tratamientos modifican la evolución de ambas condiciones se explicara también la base fisiopatológica de las mismas a nivel molecular, lo cual aporta un conocimiento de gran importancia para el abordaje de ambas condiciones en la práctica urológica diaria.

\section{STUI: Factor de Riesgo para disfunción eréctil?}

Al hablar de los factores de riesgo de la DE llama la atención que en algunas publicaciones se menciona a la sintomatología del tracto urinario inferior asociada a la HBP como uno de ellos. Pero,

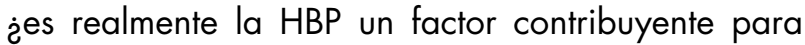
el desarrollo de la DE? Para aclarar este tema se debe estudiar cuales son las bases fisiopatológicas que condicionan una alteración de la fisiología de la erección normal del pene, tema que se aborda en otra sección de esta publicación.

Estudios en diferentes países como España (1) y Alemania (2) ponen de manifiesto una relación cuando menos epidemiológica entre la DE y la STUI. En el estudio español, Epidemiología de la DE masculina, se analizaron y ajustaron por edad los factores de riesdo de DE y para la enfermedad prostática se encontró una OR de 2.67 (Tabla I).

En 2009 Fernández et al (3) publica la prevalencia de STUI entre 1804 varones de 40 o más años de edad y evalúa los factores de riesgo para el desarrollo de la sintomatología asociada a la HBP. Se evidencia en esta muestra una prevalencia de 16,6\% de STUI siendo el riesgo tres veces mayor para sufrir clínica severa (IPSS mayor de 20) para los hombres mayores de 70 años.

En 2008 un estudio Iraní (4) correlacionó el score obtenido en el IPSS con el resultado de el IIEF. Se demostró una asociación estadísticamente significativa directamente proporcional entre la severidad de los STUI según la clasificación del IPSS en leve (07 puntos) moderada (8-19) y severa (mayor o igual a 20) y resultados desfavorables en el IIEF. Otros factores que se encuentran asociados en este y otros estudios son la edad, la obesidad, la diabetes mellitus y la dislipidemia.

No existe evidencia que el crecimiento prostático actúe directamente en la fisiopatología de la DE mediante la compresión de los nervios cavernosos afectando directamente la erección del pene.

Múltiples estudios en diferentes países han demostrado una asociación directa entre la edad y la severidad de los STUI y de la edad y DE, sin embargo la asociación directa entre STUI y DE no está clara. 
Cabe destacar que ambas condiciones se presentan con mayor intensidad en relación directamente proporcional a la edad de los pacientes. La HBP estimada para hombres de 40 años es de $40 \%$ y aumenta a $80 \%$ para hombres de 80 años de edad (5). La disfunción eréctil de cualquier etiología fue estimada por Pearlman y Kobashi en el 1972 (6) y Feldman et al en 1994 (7) siendo de $40 \%$ a los 40 años aumentando hasta cifras de $70 \%$ en hombres más de 70 años con un aumento progresivo del porcentaje de disfunción para cada decil. No se ha encontrado en esta revisión ninguna publicación que demuestre una relación directa entre la severidad de la obstrucción del tracto de salida de la orina medida por uroflujometría y el aumento de los porcentajes de disfunción eréctil con independencia de la edad.

Los síntomas del tracto urinario inferior también se han correlacionado en varios estudios con disfunciones sexuales de otro tipo distinto a la rigidez de la erección, como son la disminución del deseo sexual y del volumen del eyaculado. Más aun se ha demostrado ampliamente que los STUI disminuyen la calidad de vida medida por cuestionarios estanda- rizados y a su vez la disminución de la calidad de vida produce un impacto significativo sobre el deseo sexual, así a menor calidad de vida menor deseo sexual (8).

Aun cuando está claro que tanto la DE como los STUI están estrechamente relacionados, no existen estudios que establezcan una relación causal entre la HBP y DE. En el estudio MSAM-7 (9) analizado por Moncada I. en 2003(10) se evidencia que de una muestra de 14000 hombres de diferentes razas y culturas con edades comprendidas entre 50 y 80 años, el porcentaje de coitos exitosos era el doble para hombres asintomáticos entre 50-60 que el de los que presentaban STUI (con IPSS mayor a 19 puntos) en el mismo grupo etario, con disminución en el numero de coitos de manera inversamente proporcional al score obtenido en el cuestionario IPSS, independientemente de la edad de los pacientes. En este estudio se demuestra claramente que las disfunciones sexuales tienen una estrecha relación con los STUI sin embargo no establece, así como ningún otro de los estudios consultados, una relación causal directa con la DE.

\section{TABLA I. FACTORES DE RIESGO ASOCIADOS A DISFUNCIÓN ERÉCTIL.}

\begin{tabular}{|c|c|c|}
\hline Enfermedad & OR I.C. OR 95\% & p-valor \\
\hline Hipertensión & $1,721,162,55$ & $0,011^{*}$ \\
\hline Diabetes & $1,981,093,60$ & $0,0289^{*}$ \\
\hline Enfermedades cardíacas & $1,380,832,30$ & 0,2697 \\
\hline Enfermedades pulmonares & $2,311,124,74$ & $0,0372^{*}$ \\
\hline Alteraciones de la circulación & $2,391,483,85$ & $0,0014^{*}$ \\
\hline Enfermedades reumáticos & $2,461,643,71$ & $0,0000^{*}$ \\
\hline Hipercolesterolemia & $1,621,042,52$ & 0,0635 \\
\hline Alergias & $1,370,752,49$ & 0,2524 \\
\hline Enfermedad prostática & $2,671,484,80$ & $0,0007^{*}$ \\
\hline Medicamentos & & $0,0104^{*}$ \\
\hline Ansiolíticos & $2,281,194,34$ & $0,0057^{*}$ \\
\hline Hipnóticos & $2,781,335,81$ & 0,0638 \\
\hline Tóxicos & $1,591,022,48$ & 0,0654 \\
\hline Fumar (>40 cig./día) & $1,621,202,18$ & \\
\hline Abuso de alcohol & & \\
\hline
\end{tabular}




\section{Fisiopatología de STUI y DE}

Una serie de hipótesis se han formulado para explicar la existencia de una fisiopatología común entre STUI y DE. En la actualidad, la relación entre STUI y disfunción sexual está apoyada por cuatro teorías, no mutuamente excluyentes, que incluyen:

a) la hiperactividad autonómica y la hipótesis de síndrome metabólico,

b) los cambios en la sintetasa de óxido nítrico / óxido nítrico (NOS / NO) de la vía guanina monofosfatasa en la próstata y el pene,

c) la activación de Rho-kinasa y la vía de la endotelina, y (d) las consecuencias fisiopatológicas de la aterosclerosis pélvica $(11,12)$.

Es posible que los STUI y la DE compartan una etiología común relacionada con las enfermedades cardiovasculares y síndrome metabólico, explicándose de este modo que ambas patologías presenten los mismos factores de riesgo, destacando la diabetes mellitus, la hiperlipidemia y la obesidad, responsables directos del daño progresivo del endotelio del tejido cavernoso, alterando la generación de óxido nítrico impidiendo la relajación del músculo liso cavernoso y modificando la cascada de acciones que provocan la vasodilatación y la erección $(13,14)$. Por otra parte las endotelinas tienen un papel importantísimo como reguladores a largo plazo del tono del músculo liso de los cuerpos cavernosos y un indudable papel en la fisiología y fisiopatología peneana $(13,14)$.

No debemos olvidar mencionar el papel de los marcadores de inflamación en el diagnóstico y pronóstico de enfermedad endotelial, especialmente ateroesclerótica. Tanto los reactantes de fase aguda como los de fase crónica están siendo considerados como potenciales marcadores diagnósticos que abran el camino de opciones preventivas. La PCR se considera predictora de enfermedad cardíaca lestudio Capture). También han sido valorados los reactantes de fase crónica, entre ellos el factor transformador del crecimiento- $\beta$ (TGF- $\beta$ ) y otras citoquinas proinflamatorias como IL-1, IL-6, factor $\alpha$ de necrosis tumoral (TNF $\alpha)(13)$.

\section{Impacto del Tratamiento de la HBP en la DE}

El tratamiento de la HBP varía en función de una serie de parámetros como son los síntomas urinarios predominantes (obstructivos vs irritativos), el tamaño de la próstata, la comorbilidad asociada, y la edad del paciente. No obstante disponemos básicamente de dos armas terapéuticas: farmacológica y/o quirúrgico.

El candidato ideal para recibir tratamiento médico es aquel que presentando síntomas urinarios que le afectan de forma desfavorable en su calidad de vida, no tiene indicaciones absolutas para cirugía.

Dada la contribución del funcionamiento sexual en el mantenimiento de la calidad de vida, en la elección del tratamiento para el paciente con HBP deben tomarse en cuenta los posibles efectos negativos sobre la función sexual. Ciertas clases de tratamiento pueden beneficiar tanto a STUI y DE. Desde una perspectiva clínica, una profunda comprensión de los efectos de diferentes modalidades de tratamiento para los STUI en la función sexual es importante para orientar a los hombres que buscan tratamiento por presentar STUI. En este campo, es más correcto la utilización disfunción sexual (DS) que engloba la disminución del deseo sexual, las alteraciones de la eyaculación, la $\mathrm{DE}$, o la combinación de estas tres entidades patológica.

Las opciones terapéuticas quirúrgicas y farmacológicas de los STUI difieren en el impacto que producen sobre la DS. El tratamiento combinado con inhibidores de la $5 \alpha$-reductasa (5 ARI) y antagonistas de los receptores alfa adrenérgicos ha sido considerado el mejor tratamiento farmacológico para reducir el riesgo de progresión de la enfermedad, ya sea en términos de crecimiento de la próstata, o de progresión de los STUI o en riesgo de complicaciones. El tratamiento farmacológico de los STUI con alfa bloqueantes se asocia con menor compromiso de DS en el paciente con HBP que la terapia combinada. Estudios controlados con placebo de 5-ARI han demostrado que la DE se presenta en el $7-8 \%$ de los hombres (con placebo, 4-5\% de los hombres), disminución del deseo sexual en el $4-6 \%$ (placebo, 2-3\%), y eyaculación anormal en el 1-2\% (placebo, <1\%) (15). Un estudio abierto ha evaluado el efecto en la función sexual del tratamiento durante un año con alfuzosina en 3076 los hombres. La alfuzosina mejora de forma significativa la puntuación total del IPSS y y en los hombres con DS presentaban mejora significativa en la rigidez de la erección, en el volumen eyaculado, y el dolor / malestar en la eyaculación $(13,16)$.

Los bloqueantes de los adrenorreceptores alA están asociados con tasas bajas de disfunción sexual y con mejoría significativa de la sintomatología urinaria. Estudios comparativos demostraron que alfuzosina (bloqueante $\alpha$ l selectivo), terazosina y doxazosina (bloqueantes no selectivos) están aso- 
ciados con menores tasas de trastornos eyaculatorios que finasteride. En contraposición, la tamsulosina (bloqueante $\alpha 1 \mathrm{~A}$ selectivo) aumenta el riesgo de eyaculación anormal. De todos los $\alpha$ bloqueantes el que se ha asociado con una mejor función sexual es la alfusozina $(15,17,18)$.

Dentro de los fármacos supresores androgénicos, un estudio demostró que la terapia con finasteride disminuyó los síntomas y el tamaño de la próstata, elevó la velocidad del flujo urinario y redujo el riesgo de cirugía y de retención urinaria aguda. Sin embargo, la intervención se asoció con incrementos notables en la incidencia de impotencia, reducción del deseo sexual, disfunción eyaculatoria y ginecomastia (18).

Actualmente, la resección transuretral de la próstata (RTUP) y la adenomectomía retropúbica son los procedimientos quirúrgicos utilizados con mayor frecuencia para el tratamiento de la HPB. Sin embargo, la disfunción eyaculatoria y la impotencia son secuelas frecuentes, de la misma forma se ha demostrado que la terapia con láser, aunque mínimamente invasiva, produce un nivel de disfunción sexual similar al asociado con la RTUP.

Por tanto, podemos concluir diciendo que las técnicas quirúrgicas provocan mayores tasas de disfunción eyaculatoria e impotencia que el tratamiento farmacológico. Antes de indicar uno u otra opción terapéutica debemos tener presente este hecho e informar al paciente para que entre todos se llegue a la mejor decisión.

\section{Tratamiento de STUI y DE}

Teniendo en cuenta la alta prevalencia y la sugerida relación fisiopatológica entre STUI y DE en los hombres mayores de 50 años parece imperativo que el tratamiento terapéutico de estas dos entidades (DE y STUI) por parte del equipo de profesionales que cuidan de la salud se realice desde un punto de vista integral. Las estrategias farmacológicas destinadas al tratamiento en común de estos dos aspectos deben ser investigados en profundidad.

Los fármacos inhibidores de la fosfodiesterasa tipo 5 (IPDE5) están considerados como la primera línea de tratamiento para la DE (19). Recientemente han aparecido evidencias científicas que parecen demostrar que el tratamiento de la DE mejora la STUI, como efecto colateral altamente beneficioso. Estos resultados han sido confirmados en estudios controlados con placebo $(20,21,22)$. Un primer ensayo, con 281 hombres con STUI moderados o graves fueron aleatorizados para recibir tadalafilo $15 \mathrm{mg}$ durante
6 semanas seguido de un aumento de la dosis a 20 mg 6 semanas más) o de placebo. El IPSS mejoró significativamente con tadalafilo $5 \mathrm{mg}$ a las 6 semanas $(2,8$ frente a 1,2 puntos, $p=0,003)$, aunque la mejora fue aún más marcado en la semana 12 con la dosis de $20 \mathrm{mg}(3,8$ vs $1,7, \mathrm{p}<0,001)$. Otro ensayo, 366 hombres con STUI moderada o grave y $\mathrm{DE}$ fueron aleatorizados para recibir sildenafilo 150 mg durante 2 semanas seguido de $100 \mathrm{mg}$ durante 10 semanas) o placebo. Al finalizar el estudio, a las 12 semanas, el IPSS se redujo significativamente con sildenafilo en comparación con placebo 16,32 vs $1,93, p<0,0001)$.

La combinación de estrategias farmacológicas con fármacos para la HBP y DE ha sido demostrada en algunos estudios que consideran las ventajas y riesgos de una terapia de combinación con a l-adrenérgico los antagonistas y los inhibidores de la PDE5. La razón para utilizar una combinación de alfuzosina y sildenafilo se basa en la convergencia de las siguientes conclusiones (23). En primer lugar, está bien establecido que STUI y DS son muy frecuentes en hombres de edad avanzada y están estrechamente relacionadas con independencia de la edad y las comorbilidades cardiovasculares, y en este enlace tiene una plausibilidad biológica. En segundo lugar, debido a esta fuerte asociación entre STUI y DS, la prescripción conjunta de inhibidores de la PDE5 y alfa bloqueantes es probable que aumente, sobre todo si tenemos en cuenta que su administración conjunta no está contraindicada. En tercer lugar, los alfa bloqueantes y los IPDE5 actúan por mecanismos de acción diferentes en el tracto urogenital, y por lo tanto pueden tener efectos sinérgicos sobre STUI y DE: Alfuzosina, mediante el bloqueo de los receptores a 1-adrenérgicos y la reducción del tono simpático en el músculo liso del pene y cuello de la vejiga y la próstata, podría aumentar el oxido nítrico (NO) y mediar la relajación que produce en el músculo liso la acción del IPDE5 (sildenafilo) con el consiguiente efecto potenciador (24).

Los alfa bloqueantes, proporcionan, en estudios controlados con placebo, un alivio rápido y sostenido de los STUI, con efectos cardiovasculares y sexuales indeseables ocasionales. Si bien estos agentes difieren en su impacto en la eyaculación, tamsulosina se asocia con una mayor incidencia de eyaculación anormal, aneyaculación, que no se debe a eyaculación retrógrada (25), no hay evidencia de presentación de informes de eventos adversos espontáneos de que estos agentes menoscaben el deseo sexual o la función eréctil en comparación con placebo. Incluso pueden mejorar la función eréctil, ya sea indirectamente a través de un la mejora de STUI, o a través de un efecto adecuado en cuerpo caver- 
noso. Estos fármacos pueden contribuir a mejorar la DE impactando la balanza entre la contracción (detumescencia) y la relajación muscular (erección) de los cuerpos cavernosos (26). Aunque existen alfa bloqueantes con excesivo efecto hipotensor que puede obstaculizar la función eréctil mediante la reducción de la presión de llenado en el pene. Estudios publicados sugieren que alfuzosina y doxazosina puede tener un efecto beneficioso sobre la función sexual (27-30).

La alfuzosina parece mejorar la función sexual, especialmente en hombres con STUI graves $(27,28)$. Los resultados en modelos experimentales con alfuzosina han demostrado un efecto relajante directo en los cuerpos cavernosos (31) y un efecto potenciador de la capacidad erectil de la apomorfina (32). Si a esto añadimos que el NO, está ampliamente reconocido como importante en la relajación de los cuerpos cavernosos del músculo liso y la vasculatura pélvica y que se han identificado receptores en la próstata humana parece que hay evidencia para que la administración conjunta de un alfabloqueante y un IPDE-5 en los pacientes que tienen STUI y DE asociados potencien o mejoren, en cierta medida, los efectos beneficiosos de cada droga administrada sola (24).

Aunque los alfa bloqueantes puede mejorar la DE administrada sola, estudios piloto sin utilización de placebo sugiere que el efecto sobre la $D E$ es modesto en comparación con la de la los inhibidores de la fosfodiesterasa tipo 5 (33) (Figura 1).

Se ha publicado la comparación en niveles de eficacia entre el uso de citrato de sildenafilo 25 mg (cuatro veces por semana), asociado o no a tamsulosina, 0,4 mg (una vez al día) en 60 varones con HBP y DE durante 8 semanas. El resultado del tratamiento evaluado mediante IPSS, IIEF, SHIM, y flujometría con medida del residuo postmiccional demuestra que el tratamiento combinado no sólo fue superior a la tamsulosina para mejorar los STUI sino que además, presentó una mejora de la función sexual similar a la del IPDE5 (34). Otro estudio aleatorizado de 28 hombres con DE que no respondieron a la monoterapia con sildenafilo $1100 \mathrm{mg} 1$ hora antes del acto sexual), demostró una mejoría significativa en la DS medida mediante el IIEF si al sildenafilo se le asociaba doxazosina (4 $\mathrm{mg}$ al día) con buena tolerancia (35). Del mismo modo, en un análisis retrospectivo de los 42 hombres con DE que no respondieron a la monoterapia con tadalafilo, la adición de un bloqueador adrenérgicos (tamsulosina $10 \mathrm{mg}$ al día) a tadalafilo (20 mg en la demanda) la presentó una mejora en la DE del $71 \%$ de los pacientes con una buena tolerabilidad cardiovascular (36). El tratamiento combinado con alfuzosina (10 mg al día) y sildenafilo $25 \mathrm{mg}$ (a demanda) también se ha demostrado como las otras combinaciones como una terapia segura, y en un estudio piloto fue la alternativa terapeútica más eficaz para mejorar tanto los STUI como la DE en pacientes con ambas patologías (37).

La introducción de estos agentes orales ha revolucionado el tratamiento de la DE pero el resto de modalidades terapéuticas -como las prótesis peneanas o las inyecciones intracavernosas de drogas vasoactivas- presentan en el momento actual subgrupos de pacientes (diabetes y enfermedad cardiovascular), que lo precisan como tratamiento. Kaplan et al (38) investigó el efecto sinérgico de la doxazosi-
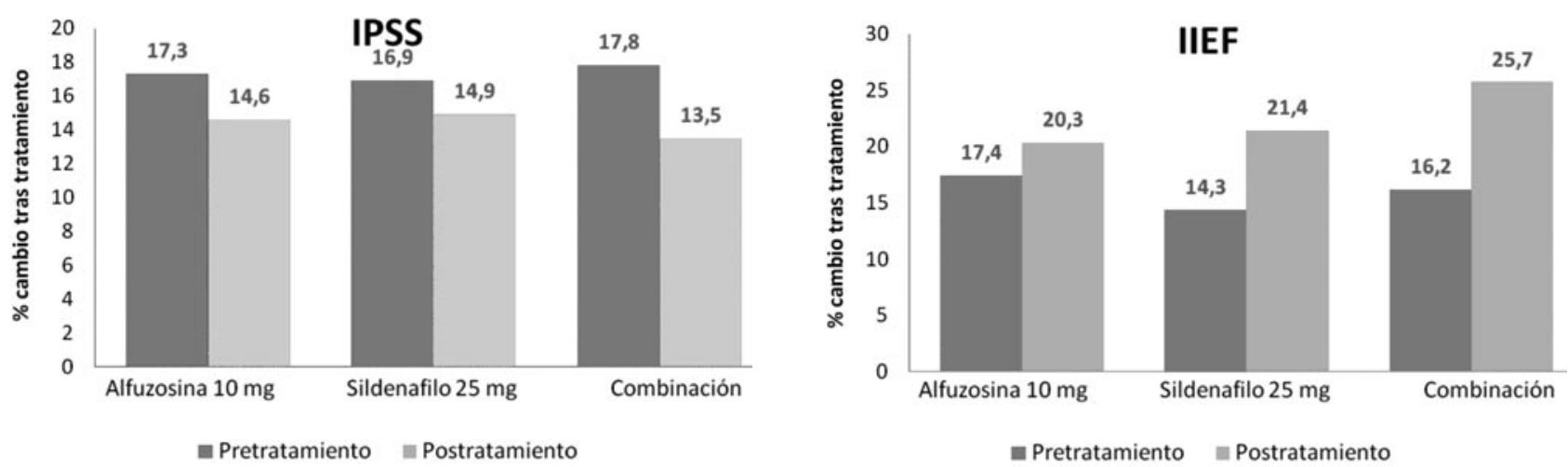

FIGURA 1. Media mejoras desde el inicio en el IPSS y el aumento en el dominio IIEF (función eréctill) en la semana 12 de tratamiento con tamsulosina $10 \mathrm{mg} O D$, OD sildenafilo $25 \mathrm{mg}$ o la combinación de ambos. Para IIEF, las puntuaciones más altas indican una mejor función. Para IPSS, bajas puntuaciones indican una mejora. 
na y la inyección intracavernosa de alprostadil (ICI) en pacientes para quienes $\mathrm{ICl}$ era el tratamiento de elección El tratamiento combinado con doxazosina mejoró la DE en esta población limitada, evaluada mediante IIEF.

A gran escala, estudios controlados con placebo son necesarios para confirmar estos datos y dilucidar el papel de la terapia de combinación para el tratamiento de estas dos condiciones.

\section{BIBLIOGRAFÍA y LECTURAS RECOMENDADAS ( ${ }^{*}$ lectura de interés $y^{* *}$ lectura fundamental)}

1. Martin-Morales A, Sanchez-Cruz JJ, Saenz de Tejada I, Rodriguez-Vela L, Jimenez-Cruz JF, Burgos-Rodriguez R Prevalence and independent risk factors for erectile dysfunction in Spain: results of the Epidemiologia de la Disfuncion Erectil Masculina Study. J Urol. 2001 Aug;166(2):569-74; discussion 574-5

2. Braun MH, Sommer F, Haupt G, Mathers MJ, Reifenrath B, Engelmann UH..Lower urinary tract symptoms and erectile dysfunction: co-morbidity or typical "Aging Male" symptoms? Results of the "Cologne Male Survey Eur Urol. 2003 Nov;44(5):588-94

3. Fernández Pérez C, Moreno Sierra J, Cano Escudero S, et al. Prevalence of lower urinary tract symptoms related with benign prostatic hyperplasia. Study of 1804 men aged 40 or older in Madrid. Actas Urol Esp. 2009; 33(1): 43-51

4. Darab Mehraban, Gholam Hossein Naderi, Seyed Reza Yahyazadeh, et al. Sexual Dysfunction in Aging Men with Lower Urinary Tract Symptoms. Urology, 2008: 5 (4): 260-264.

5. Berry SJ, Coffey DS, Walsh PC, et al. The development of benign prostatic hyperplasia with age. $\mathrm{J}$ Urol. 1984; 132: 474-479

6. Feldman HA, Goldstein I, Hatzichristou DG, Krane RJ, Mckinlay JB. Impotence and its medical and psychosocial correlates: results of theMassachusetts Male Aging Study. J Urol. 1994; 151:54-61.

7. Pearlman CK, Kobashi LI. Frequency of intercourse in men. J Urol. 1972; 107:298-301.

8. Roberts RO, Jacobsen SJ, Rhodes T, et al. Natural history of prostatism: impaired health states in men with lower urinary tract symptoms. J Urol. 1997; 157:1711-1717.

9. Rosen R, Altwein J, Boyle P, et al. Lower urinary tract symptoms and male sexual dysfunction: the multinational survey of the aging.male (MSAM7) Prog Urol. 2004; 14(3): 332-44.
**10. Moncada I. LUTS: A Risk Factor for Sexual Dysfunction in the BPH Patient. European Urology Supplements. 2003; 2:3-8.

11. McVary K. Lower urinary tract symptoms and sexual dysfunction: epidemiology and pathophysiology. BJU Int 2006; 97(2): 23-8.

12. McVary KT, Rademaker A, Lloyd GL, et al. Autonomic nervous system overactivity in men with LUTS secondary to benign prostatic hyperplasia. J Urol 2005; 174: 1327.

13. García Cardoso J.V., López Farré A., Vela Navarrete R. Disfunción eréctil: papel del laboratorio en la evaluación diagnóstica y pronóstica. Actas Urol.Esp. 2005; 890-898.

14. Martin Morales A, Sánchez Cruz J, Saenz de Tejada I, et al. Prevalence and independent risk factors for erectile dysfunction in Spain: Results of the epidemiology of the erectile dysfunction study". J Urol, 2001; 166 (2); 569-575.

*15. Martín-Morales A, Meyer G, Ramírez E. Prevalencia de disfunción eyaculatoria secundaria al tratamiento con alfa-bloqueantes en pacientes con hiperplasia benigna de próstata. Actas Urol Esp. 2008; 32 (7): 705-712.

16. Lepor H, Williford WO, Barry MJ, et al. The impact of medical therapy on bother due to symptoms, quality of life and global outcome, and factors predicting response. J Urol, 1998; 160: 1358- 1367.

17. Hernández Fernández C, Castaño González I, Moncada Iribarren I, Jara Rascón J, Moralejo Gárate M. Tratamiento con doxazosina en 3347 pacientes con síntomas del tracto urinario inferior. Efecto sobre su función sexual. Estudio impros. Actas Urol. Esp.2004; 290-297.

**18. Moorselaar VJ. LUTS and sexual dysfunction: Implications for management of BPH. Eur Urol Supplements. 2003; 2:13-2.

19. Uckert S, Oelke M, Stief CG, et al. Immunohistochemical distribution of cAMP- and cGMP-phosphodiesterase (PDE) isoenzymes in the human prostate. Eur Urol 2006; 49: 740-5.

20. Sairam K, Kulinskaya E, McNicholas T, et al. Sildenafil influences lower urinary tract symptoms. BJU Int 2002; 90: 836-9.

*21. Mulhall JP, Guhring P, Parker M, et al. Assessment of the impact of sildenafil citrate on lower urinary tract symptoms in men with erectile dysfunction. J Sex Med 2006; 3: 662-7.

22. Roehrborn CG, McVary KT, Kaminetsky JC, et al. The efficacy and safety of tadalafil administered once a day for lower urinary tract symptoms (LUTS) in men with benign prostatic hyperplasia (BPH). J Urol 2006; 175 :527.

23. Heaton J. LUTS and Sexual Dysfunction: What is the Link and How Can it Be Managed? Eur Urol Supplements. 2006; 5 :722-728. 
24. Van Moorselaar RJ, Hartung R, Emberton M, et al, for the ALF-ONE Study Group. Alfuzosin $10 \mathrm{mg}$ once daily improves sexual function in men with lower urinary tract symptoms and concomitant sexual dysfunction. BJU Int 2005; 95: 603-8.

25. Chapple CR, Wyndaele JJ, Nordling J, et al. Tamsulosin, the first prostate-selective alpha1Aadrenoceptorantagonist: a meta-analysis of two randomised, placebo-controlled, mulitcentre studies in patients withbenign prostatic obstruction (symptomatic BPH). Eur Urol 1996; 29: 155-67.

26. Lepor H. Long-term evaluation of tamsulosin in benign prostatic hyperplasia: placebo-controlled, double-blind extension of phase III trial. Tamsulosin Investigator Group. Urology 1998; 51: 901-6.

27. Roehrborn CG, for the ALFUS Study Group. Efficacy and safety of once-daily alfuzosin in the treatment of lower urinary tract symptoms and clinical benign prostatic hyperplasia: a randomized, placebo-controlled trial. Urology 2001; 58: 953-9.

28. Hartung R, Matzkin H, Alcaraz A, et al. Age, comorbidity and antihypertensive comedication do not affect cardiovascular tolerability of $10 \mathrm{mg}$ alfuzosin once daily. J Urol 2006; 175: 624-8.

29. Elhilali M, Emberton M, Matzkin H, et al., for the ALF-ONE Study Group. Long-term efficacy and safety of alfuzosin $10 \mathrm{mg}$ once daily: a 2-year experience in 'real-life' practice. BJU Int 2005; 97: 513-9.

30. McConnell JD, Roehrborn CG, Bautista OM, et al. The longterm effect of doxazosin, finasteride, and combination therapy on the clinical progression of benign prostatic hyperplasia. $\mathrm{N}$ Engl $\mathrm{J}$ Med 2003; 349: 2387-98.
31. Palea S, Barras M. Comparison of the relaxant effects of alfuzosin, phentolamine and sildenafil on rabbit isolated corpus cavernosum. BJU Int 2003; 91: 873-7.

32. Mayoux E, Ramirez JF, Pouyet T, et al. Alfuzosin improves penile erection triggered by apomorphine in spontaneous hypertensive rats. Eur Urol 2004; 45:110-6.

33. Sciarra A. Lower Urinary Tract Symptoms (LUTS) and Sexual Dysfunction (SD): New Targets for New Combination Therapies? Eur Urol, 2007; 51: 1485-1487.

34. Tuncel A, Nalcacioglu V, Ener K, et al. Comparison of efficacy of sildenafil-only, sildenafil plus tamsulosin, and tamsulosin - only in treating lower urinary tract symptoms and erectile dysfunction. Eur Urol Supplements. 2008; 7 (3): 171.

35. Carson CC. Combination of phosphodiesterase5 inhibitors and alpha-blockers in patients with benign prostatic hyperplasia: treatments of lower urinary tract symptoms, erectile dysfunction, or both? BJU Int, 2006; 97(2):39-43.

36. Rosen R. Update on the relationship between sexual dysfunction and lower urinary tract symptoms/benign prostatic hyperplasia. Current Opinion in Urology 2006; 16:11-19.

37. Kaplan SA, Gonzalez RR, Te AE. Combination of alfuzosin and sildenafil is superior to monotherapy in treating lower urinary tract symptoms and erectile dysfunction. Eur Urol, 2007; 51:1717-23.

38. Kaplan SA, Reis RB, Kohn IJ. Combination therapy using oral alpha-blockers and intracavernosal injection in men with erectile dysfunction. Urol, 1998; 52 (7): 39-43. 